\title{
Disseminated Kaposi's Sarcoma as Initial Event in AIDS
}

\author{
Clarice Jordão, Erica Paulina de Barros, Beatriz Moritz Trope and Marcia Ramos-e-Silva*
}

Sector of Dermatology, University Hospital and School of Medicine, Federal University of Rio de Janeiro, Brazil

\begin{abstract}
The authors report a case seen in the internal medicine infirmary, which was diagnosed as acquired immunodeficiency syndrome (AIDS) because of cutaneous and visceral Kaposi's sarcoma (KS) involvement. They highlight the importance of the dermatologist in the multidisciplinary care and approach some etiopathogenical and therapeutical features of KS. Chemotherapy with paclitaxel together with antiretroviral therapy (HAART) is described by the authors because of the good therapeutic outcome achieved.
\end{abstract}

\section{Keywords: AIDS; HIV; Kaposi's sarcoma; HAART}

\section{Introduction}

KS is a multicentric angioproliferation definer of AIDS. In general it occurs in the context of progression of the immunosuppression, but it can present as an initial manifestation, involving not only the skin but also internal organs.

\section{Case Report}

A white married man, born and living in Rio de Janeiro, was admitted at the internal medicine infirmary of the University Hospital of the Federal University of Rio de Janeiro for investigation of a cervical lymphodenomegaly, afternoon fever, and weight loss initiated a few months before.

At examination intense facial edema, more pronounced on the right side; purple macula in the upper right eyelid region, erythematousviolaceous conjunctival lesion, purple plaque in the hard palate, visible and palpable lymphnodes in the cervical, axillary and inguinal chains, and erythematous-violaceous lesions with poorly defined contours in penis glans and shaft were observed (Figures 1-3).

During hospitalization, the patient was evaluated by several specialties, including Dermatology. A biopsy of the cervical lymph node and serology for HIV were requested for investigation of the consumptive syndrome. Thorax, abdomen and pelvis tomography were also carried out as well as upper intestinal endoscopy and rectal sigmoidoscopy.

The diagnostic hypothesis of disseminated epidemic KS was first suggested by the Dermatologists that consulted the patient in the infirmary. It was confirmed later by positive HIV serology (CD4: 174 cells $/ \mathrm{mm}^{3}$; viral load: above the maximum limit) and the cervical lymphnode histopathology showing KS. The remaining complementary exams carried out for assessment of systemic involvement demonstrated gastroduodenal KS, in addition to petechiae in the rectum.

TARV (stavudine, lamivudine, efavirenz), sulfamethoxazole/ trimethoprim $(160 \mathrm{mg} / 800 \mathrm{mg})$ and chemotherapy with paclitaxel $\left(100 \mathrm{mg} / \mathrm{m}^{2}\right.$ of body surface every two weeks) were initiated and an excellent therapeutic response was already observed at the eighth week of treatment with visible reduction of the cervical lymphodenomegaly, skin and mucosal lesions, and disappearance of the afternoon fever.

\section{Discussion}

Kaposi's sarcoma is an angioproliferative multicentric disorder of cells of endothelial origin, described in 1872 by the Hungarian dermatologist Moritz Kaposi [1,2].
Four variants of KS are referred: classic, endemic, associated to immunosuppression/transplant and endemic/associated to HIV. Despite all forms sharing similar histological characteristics, they differ regarding epidemiology, morphotopography and progression course.

Classical KS was described in Western Europe and the Mediterranean Area with preference for men with an estimated prevalence of 15:1 [2]. The lesions present as plaques or firm, erythematous-violaceous nodes, located preferably in the lower limbs, with possibility to progress to the upper limbs within years or decades. Visceral or mucous involvement occurs in $10 \%$ of cases; with possibility of lymphedema of the lesions locally compressing lymphatic structures.

The endemic form was reported in Africa in the 50's. With growing incidence, it affects children and young adults with variable clinical presentation, such as indolent cutaneous lesions, infiltrative lesions located on the limbs and aggressive forms with visceral involvement. Generalized lymphadenopathy is a common presentation in children. Endemic KS generally has an aggressive behavior with poor response to therapy [3].

When associated to immunosuppression/transplant an average interval ranging from 29 to 31 months is observed after the transplant of the solid organ to the diagnosis of the disease [4,5]. This is a form that tends to be aggressive, involving lymph nodes, mucosa and even internal organs which may occur in approximately half of the cases. In this clinical form, KS lesions may occur in extra cutaneous sites with or without presence of lesions in the skin [2].

In 1981, Friedman-Kien studied KS lesions in young homosexual males [6]. This variant was related to AIDS, a recently discovered disease at the time. Clinically it presents with reddish or asymptomatic violet lesions which may evolve to nodules with eventual ulceration. Mucous involvement is described in up to $30 \%$ of the cases. Impairment of lymph drainage may occur, determining variable local edema. In

*Corresponding author: Marcia Ramos-e-Silva, Associate Professor and Head, Sector of Dermatology, University Hospital and School of Medicine, Federal University of Rio de Janeiro, Rua Dona Mariana 143/C-32, 22280-020 Rio de Janeiro, Brazil, Tel: 55-21-22864632l; E-mail: ramos.e.silva@dermato.med.br

Received March 11, 2015; Accepted March 31, 2015; Published April 11, 2015

Citation: Jordão C, de Barros EP, Trope BM, Ramos-e-Silva M (2015) Disseminated Kaposi's Sarcoma as Initial Event in AIDS. J AIDS Clin Res 6: 450 doi:10.4172/2155-6113.1000450

Copyright: (C) 2015 Jordão C, et al. This is an open-access article distributed under the terms of the Creative Commons Attribution License, which permits unrestricted use, distribution, and reproduction in any medium, provided the original author and source are credited. 


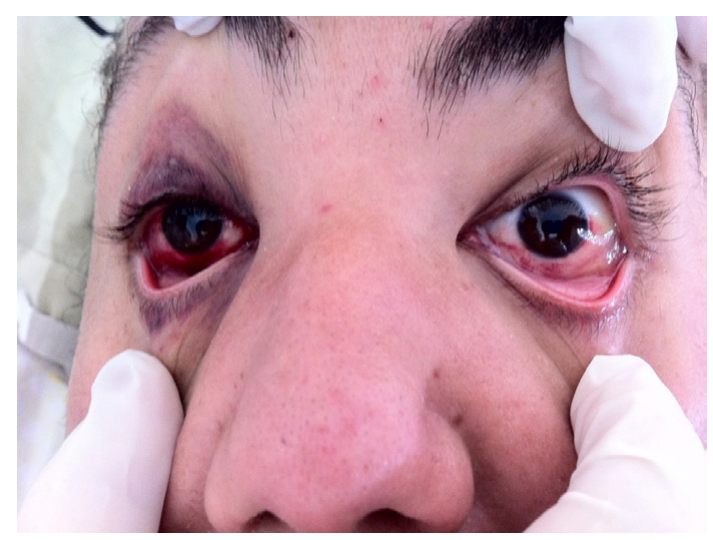

Figure 1: Erythemato-violaceous macules on the conjunctiva and eyelids.

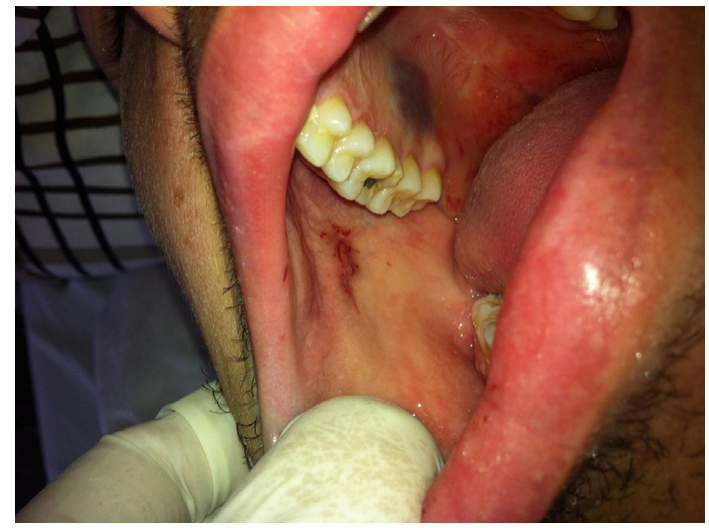

Figure 2: Erythemato-violaceous macule on the buccal mucosa.

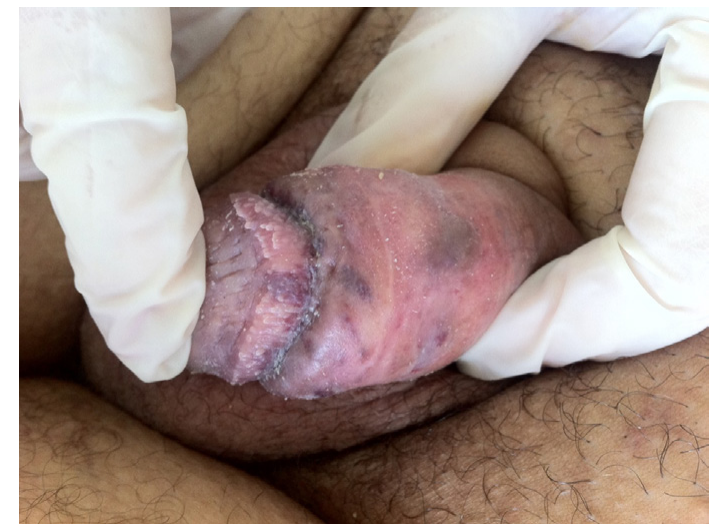

Figure 3: Erythemato-violaceous macules on the penis (PPP also observed)

epidemic KS there is also the presence of isolated or disseminated cutaneous lesions with or without involvement of internal organs [2].

In 1994, Chang et al. identified DNA fragments of the virus of the Herpesviridae family, which would be related to KS, also known as Herpesvirus 8 (HHV8) or Herpesvirus associated to KS (KSHV). This virus was found in over $95 \%$ of KS lesions regardless of the site involved or clinical subtype. The presence of HHV8 seems to be the primary and necessary factor for tumor development [7]. It is postulated that this virus would create an angiogenic inflammatory condition for appearance of the KS lesions [8]. HHV8 infects B-lymphocytes and coexists with the host without causing any disease, establishing a latent infection, without production of viral particles. It expresses proteins and non-codifying RNA that promotes cellular proliferation. Normally the immune system limits such proliferation, but if the process fails, the disease may establish itself [9]. The capacity of the disease to progress is directly related to immunological factors of the host [2]

In the tumoral environment there are low TGF- $\beta$ levels and high levels of IL-6 and VEGF, as well as other factors of pro-inflammatory growth, chemokines and interleukins, interferon $\gamma$ and IL-10. Among the oncogenic viral proteins, the latency of the inhibiting protein FLICE viral (v-FLIP) was recently indicated as the molecular interrupter of KS Genesis induced by HHV8. The latency of the nuclear viral antigen (LANA), codified by gene ORF73 of HHV8, may cause a deregulation directly increasing the rate of clonal proliferation of the cells infected by HHV8 [10] .

KS lesion histopathology reveals proliferation of fibroblasts and endothelial cells. There is also presence of vascular structures in clefts with overflow of red blood cells, deposition of hemosiderin and later presence of fusiform cells. These cells are positive for vimentin, and in the initial phase, can also be positive for factor VIII and CD30 [8]. Immunohistochemistry of the LANA-1 antigen of HHV8 can be helpful in difficult cases [11].

At the time of the diagnosis, it is necessary to perform a clinical laboratorial staging assessment. This includes: complete physical skin examination, as well as of the oral and genital mucosa, lymph nodes palpation, chest X-Ray, and, in cases of visible mucous involvement, abdominal ultrasound, upper digestive endoscopy and colonoscopy [8]

Treatment of KS will depend on the lesion extension and location and clinical progress of the disease. For solitary lesions or in small number, we have some therapeutic possibilities, such as: surgical excision, laser therapy, cryotherapy, alitretinoin and radiotherapy [8].

KS lesions are radiosensitive with a regression rate of $80 \%$ to $90 \%$, with radiotherapy indicated only for selected cases, due to the high rate of morbidity of the collateral effects [8].

For forms associated to HIV, introduction of an antiretroviral scheme is mandatory for correction of the underlying immunosuppression. Improvement of the immune profile usually causes total or partial remission of the KS lesions, by increasing the level of antibodies directed against viral protein $\mathrm{k} 8.1$ and by reestablishing the response by T CD8 against epitopes of HHV8 [12].

Systemic chemotherapy is indicated for multiple lesions and in cases with visceral involvement. Some authors indicate it in case of facial or palmoplantar involvement [8]. Chemotherapy is described with vinblastine with or without bleomycin, liposomal doxorubicin, Interferon $\alpha$ or paclitaxel [13].

Paclitaxel is a powerful anti-tumor agent, used for treatment of ovary, breast and lung carcinomas. The drug derives from a tree found in the Pacific whose action mechanism is on the tubulin. By stabilizing the microtubules, it affects cell processes in non-mitotic phases, this way inhibiting the growth of tumors of fast or slow proliferation. The authors suggest that this chemotherapy approach should be the first choice in seriously ill patients, and as second choice, after therapeutic failure with interferon $\alpha$ and/or vinblastine [13].

Several studies assess possible predictor response factors to the treatment, such as CD4 cell count, viral load, number of DNA copies of HHV8, without any of them consistently identified [14]. 


\section{Conclusion}

The appearance of KS coincides with a deterioration of the immunologic status of the HIV bearer, arising usually in more advanced immunosuppression stages $\left(\mathrm{CD} 4<200 \mathrm{cells} / \mathrm{mm}^{3}\right)$. With introduction of HAART, a reduction of the number of reported KS cases was found; however more recently several cases have been notified, including some with very aggressive clinical presentation.

The relevance of a fast recognition of the disease and its stage needs to be stressed in order to begin the treatment as soon as possible, having in view that the disease has a high rate of mortality, especially when it presents in such an exuberant form as that of the patient presented.

Our objective is to highlight the participation of the dermatologist in a diagnostic investigation within a multidisciplinary context in a general Hospital. The cutaneous and mucous lesions were initially attributed to thrombocytopenia and were underestimated, for having acted as signalers for the dermatologic diagnosis of KS.

What also called our attention in the HAART era was the fact that we were making an AIDS diagnosis in a patient who had KS as the initial event. Frequently we observe patients with epidemic KS with localized lesions, but in this case we were surprised by the extension of the clinical picture.

Both from the individual, as from the public health standpoint, it is not desirable that an AIDS diagnosis should have KS as defining disease, and, despite the seriousness of the picture, the therapeutic response presented by our patient was surprisingly favorable.

\section{References}

1. Uldrick TS, Whitby D (2011) Update on KSHV epidemiology, Kaposi Sarcoma pathogenesis, and treatment of Kaposi Sarcoma. Cancer Lett 305: 150-162.

2. Antman K, Chang Y (2000) Kaposi's sarcoma. N Engl J Med 342: 1027-1038.
3. Fatahzadeh M (2012) Kaposi sarcoma: review and medical management update. Oral Surg Oral Med Oral Pathol Oral Radiol 113: 2-16.

4. Montagnino G, Bencini PL, Tarantino A, Caputo R, Ponticelli C (1994) Clinica features and course of Kaposi's sarcoma in kidney transplant patients: report of 13 cases. Am J Nephrol 14: 121-126.

5. Lesnoni La Parola I, Masini C, Nanni G, Diociaiuti A, Panocchia N, et al. (1997) Kaposi's sarcoma in renal-transplant recipients: experience at the Catholic University in Rome, 1988-1996. Dermatology 194: 229-233.

6. Friedman-Kien AE (1981) Disseminated Kaposi's sarcoma syndrome in young homosexual men. J Am Acad Dermatol 5: 468-471.

7. Chang Y, Cesarman E, Pessin MS, Lee F, Culpepper J, et al. (1994) Identification of herpesvirus-like DNA sequences in AIDS-associated Kaposi's sarcoma. Science 266: 1865-1869.

8. Potthoff A, Brockmeyer NH (2007) HIV-associated Kaposi sarcoma: pathogenesis and therapy. J Dtsch Dermatol Ges 5: 1091-1094.

9. Cesarman E (2011) Gammaherpesvirus and lymphoproliferative disorders in immunocompromised patients. Cancer Lett 305: 163-174.

10. Riva G, Barozzi P, Torelli G, Luppi M (2010) Immunological and inflammatory features of Kaposi's sarcoma and other Kaposi's sarcoma-associated herpesvirus/human herpesvirus 8-associated neoplasias. AIDS Rev 12: 40-51.

11. Patel RM, Goldblum JR, Hsi ED (2004) Immunohistochemical detection of human herpes virus-8 latent nuclear antigen-1 is useful in the diagnosis of Kaposi sarcoma. Mod Pathol 17: 456-460.

12. El Amari EB, Toutous-Trellu L, Gayet-Ageron A, Baumann M, Cathomas G et al. (2008) Predicting the evolution of Kaposi sarcoma, in the highly active antiretroviral therapy era. AIDS 22: 1019-1028.

13. Kim SY, Kim DH, Lee HJ, Seo YJ, Lee JH, et al. (2011) Treatment of Disseminated Classic Type of Kaposi's Sarcoma with Paclitaxel. Ann Dermatol 23: 504-507.

14. Nguyen HQ, Magaret AS, Kitahata MM, Van Rompaey SE, Wald A, et al. (2008) Persistent Kaposi sarcoma in the era of highly active antiretroviral therapy: characterizing the predictors of clinical response. AIDS 22: 937-945 